Retrospective Study

\title{
Clinical Effectiveness of Percutaneous Adhesiolysis Using Navicath for the Management of Chronic Pain Due to Lumbosacral Disc Herniation
}

\author{
Jung Hwan Lee, MD, PhD and Sang-Ho Lee, MD, PhD
}

From: Wooridul Spine Hospital, Seoul, Korea

Dr JH Lee is with the Department of Physical Medicine and Rehabilitation, Wooridul Spine Hospital, Seoul, Korea

Dr. SH Lee is with the Department of Neurosurgery, Wooridul Spine Hospital, Seoul, Korea

Address correspondence: Juan Hwan Lee, MD Wooridul Spine Hospital 46-17 chungdamdong gangnamgu Seoul, Korea E-mail:

j986802@hanmail.ne

Disclaimer: This study was supported by Wooridu

Spine Foundation. Conflict of interest: None.

Manuscript received: $12 / 14 / 2011$

Revised manuscript

received: 02/26/2012 Accepted for publication: $03 / 22 / 2012$

Free full manuscript: www.painphysicianjournal.
Background: Epidural steroid injection has been frequently performed to treat chronic pain due to lumbosacral disc herniation (L-HIVD). However, a considerable number of patients do not achieve pain relief using this method because perineural or epidural adhesions prevent the spread of injectate into the epidural space. Percutaneous adhesiolysis (PA) is thought to be a useful method because it can eliminate the deleterious effects of adhesion.

Objectives: This study aimed to evaluate the effectiveness of PA in managing chronic pain due to L-HIVD and the clinical and radiological predictive factors for the effectiveness of PA using NaviCath ${ }$.

Study design: Retrospective study

Setting: Spine hospital

Methods: From a group of patients diagnosed with L-HIVD, we selected the 86 patients who underwent PA with NaviCath who had experienced chronic lower back or leg pain for at least 3 months and had failed to respond to anti-inflammatory medications or physical therapy of at least 1 month's duration and fluoroscopy guided transforaminal epidural injection. We recorded the Numeric Rating Scale for back pain (NRS back) and leg pain (NRS leg) and the Oswestry Disability Index (ODI) at pretreatment, 2 weeks, and 3 months after treatment. Clinical data and magnetic resonance imaging (MRI) findings were obtained to assess the possible predictive factors for PA efficacy.

Limitations: Retrospective chart review without a control group.

Results: At 2 weeks after PA, significant improvement was observed in NRS back, NRS leg, and ODI compared with pretreatment. This improvement was maintained until 3 months after treatment. Among 86 patients, 61 (70.9\%), 53 (61.6\%) and 61 patients (70.9\%) showed successful outcomes in NRS back, NRS leg, and ODI at 2 weeks, respectively. Among 74 patients who were followed up at 3 months, $47(63.5 \%), 44$ patients $(59.5 \%)$, and 50 patients $(67.6 \%)$ showed successful results in NRS back, NRS leg, and ODI at 3 months, respectively.

A significantly higher proportion of patients with a history of previous lumbar surgery showed unsuccessful results on NRS back, NRS leg, and ODI scores at 2 weeks and 3 months. Co-existence of spinal stenosis was associated with a significantly higher proportion of unsuccessful results in NRS back and ODI at 2 weeks and 3 months, as well as NRS leg at 3 months. Patients with spondylolisthesis also showed a significantly higher proportion of unsuccessful results in NRS and ODI at 2 weeks.

Conclusion: PA with NaviCath showed clinical effectiveness in the treatment of chronic pain due to L-HIVD that was not responsive to transforaminal epidural injection. Previous surgery and the presence of spinal stenosis or spondylolisthesis were poor prognostic predictors. This procedure may enable the physician to place the catheter tip and deliver medicine more precisely.

Key words: Percutaneous adhesiolysis, lumbosacral disc herniation, Numeric Rating Scale, Oswetry Disability Index

Pain Physician 2012; 15:213-221 


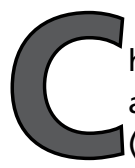
hronic lower back or radiating leg pain from a lumbosacral herniated intervertebral disc (L-HIVD) is a common condition. Its prevalence ranges from $35 \%$ to $75 \%$ at 12 months after the initial onset of symptoms (1). Chronic lower back or leg pain was found to occur not only in response to mechanical stimuli, but also to chemical irritation around the nerve root sheath and sinuvertebral nerve (2). Leakage of the disc material into the epidural space following an annular tear leads to acute inflammation and consequent fibrocyte deposition and epidural adhesions, which result in compression of the nerve roots (3-6). While peridural or neural fibrosis in themselves are not painful, they can produce pain by trapping spinal nerves so that movement produces tension in the inflamed nerves $(2,3)$. Generally, fluoroscopy-guided epidural injections have been used to treat chronic pain due to L-HIVD. Through their anti-inflammatory action clinical efficacy has been obtained $(7,8)$. Transforaminal epidural injections have produced favorable results for managing lumbosacral radicular pain secondary to L-HIVD and spinal stenosis $(9,10)$. Caudal epidural injections have provided functional improvement and pain relief for patients with chronic low back pain due to postsurgery syndrome $(11,12)$. However, a considerable number of patients do not achieve meaningful pain relief through epidural injections. One study showed that among patients undergoing transforaminal or caudal epidural injection, only one-third obtained more than 2 months of pain relief (13). Another study revealed that epidural injection had better short-term results than interspinous or intramuscular injection, but this benefit was not maintained for up to 6 weeks in patients with L-HIVD or spinal stenosis (14). A retrospective study reported that although approximately $50 \%$ of patients with radicular symptoms may receive temporary relief, long-term relief occurs in less than $25 \%$ of patients (15). This was because the epidural space in these cases was restricted by perineural or epidural adhesions/ fibrotic tissues, and the injectate frequently failed to spread effectively into the ventral epidural space (16).

Percutaneous adhesiolysis (PA) is a minimally invasive therapy in which a catheter is placed directly into the herniated disc or scar tissue compromising the nerve root. It has potential as a useful treatment method for patients with chronic pain that is refractory to conservative treatments (17). The rationale for PA is that chronic pain is mainly caused by perineural fibrosis and that PA has the ability to eliminate the deleterious effects of adhesion, which can physically pre- vent the direct application of drugs around the nerves. As a result, PA ensures the delivery of high concentrations of injected drugs to the target areas $(16,18-21)$. PA has produced clinical benefits in patients who have failed to respond to conservative treatment, including fluoroscopy-guided epidural injections (22). According to a comparative study between PA and caudal steroid injection in postsurgery syndrome, PA obtained significantly better clinical efficacy than caudal injection (23). To the best of our knowledge, all previous literature about PA was about using a Racz catheter. Whereas the catheter and spring tip on the Racz catheter cannot be steered, NaviCath (Myelotec Inc, Roswell, GA) has a steerable catheter and an atraumatic tip. This property enables the physician to place the catheter tip and deliver pain medication more precisely around the nerve root sheath and perform mechanical lysis. In this study, we intended to evaluate the clinical efficacy of PA with NaviCath in a clinical setting. Epidural injection is more often performed than PA since epidural injection is a simpler and less expensive procedure. However, PA is considered for the patient who is refractory to epidural injection. We thought the study, which was clinically informative and applicable, would be more implicative. Therefore, we aimed to evaluate PA efficacy in patients who were unresponsive to transforaminal epidural injections, instead of comparing the clinical efficacy between PA and epidural injection.

The purpose of this study was to evaluate the effectiveness of PA with NaviCath for managing chronic lower back and leg pain in patients with L-HIVD for whom transforaminal epidural injections were not successful. The study also investigated the clinical and radiological factors that are related to the clinical effectiveness of PA.

\section{Methods}

\section{Materials}

Informed consent was obtained by the Institutional Review Board of Wooridul Spine Hospital. From a group of patients diagnosed with L-HIVD, we selected the patients who underwent PA with NaviCath. These patients had experienced chronic lower back or leg pain for at least 3 months and had failed to respond to anti-inflammatory medications or physical therapy of at least one month and fluoroscopy-guided transforaminal epidural injection. We defined the failure of transforaminal epidural injection as the absence of 
a $50 \%$ or more reduction of the Numeric Rating Scale (NRS) compared to preinjection, when at least 2 injections had been administered over 2 months. Finally, 86 patient charts were reviewed.

\section{Methods}

\section{Data collection}

We obtained clinical data such as age, sex, duration of symptoms in months, predominant symptom (axial back pain versus radiating leg pain) and a history of previous lumbar surgery. Magnetic resonance imaging (MRI) findings that were recorded included the type and location of the herniated disc, number of lesion levels (single versus multiple levels), grade of nerve root compression, and the co-existence of spinal stenosis. The type of herniated disc was classified as bulging, protrusion, extrusion, or sequestration. The location of the herniated disc was classified as central, subarticular, foraminal, or extraforaminal disc herniation (24).

\section{Clinical evaluation}

The NRS for back pain (NRS back) and leg pain (NRS leg) as well as the Korean version of the Oswestry Disability Index (ODI) were used to evaluate the clinical effectiveness in terms of pain reduction and functional improvement at pretreatment, 2 weeks, and 3 months after treatment (25). The NRS represented no pain with 0 and the worst pain imaginable with 10 (26). All patients were asked to give their answers considering the average severity of their symptoms over the last one-week period (27). The Korean version of ODI was utilized for functional assessment that ranged from 0 to 50 . The value and validity of the NRS and Korean version of ODI have been reported previously $(20,25,28)$. Successful pain relief was described as a $50 \%$ or more reduction of the NRS, and successful functional improvement was defined as a $40 \%$ or more reduction of the ODI (23).

\section{Percutaneous adhesiolysis}

PA was performed under fluoroscopy in a sterile operating room with monitoring equipment for blood pressure, pulse rate, and pulse oximetry. The fluoroscopy was adjusted over the lumbosacral area such that the caudal approach could be used in both the anteroposterior and lateral views. After the appropriate positioning of fluoroscopy, the needle insertion area was determined around the sacral hiatus and was injected with local anesthetics. A tiny incision was made at the needle insertion area and a 15-gauge Tuohy needle with an introducer was inserted into the epidural space through the sacral hiatus. An epidurogram was obtained after injecting approximately 2 to $5 \mathrm{~mL}$ of contrast media to confirm that the needle was placed in the epidural space and to avoid intravascular or subarachnoid needle placement. A NaviCath was passed through the introducer after removal of the Tuohy needle under fluoroscopic visualization, and at least $5 \mathrm{~mL}$ of contrast media were injected to identify the filling defects by examining the contrast flow into the nerve roots. The catheter was positioned near the filling defect and the suspected pain source area. Subsequently, adhesiolysis and decompression were carried out by distension with normal saline and by mechanical means using the catheter. When the catheter was placed in the suspected pain area, some patients indicated that they felt pain similar to what they had been suffering from. After adhesiolysis, approximately $3 \mathrm{~mL}$ of contrast media were injected in order to confirm that satisfactory filling was obtained epidurally and at the targeted nerve root without subarachnoid or intravascular flow. Then, a mixture of $4 \mathrm{~mL}$ of $1 \%$ lidocaine and $40 \mathrm{mg}$ of triamcinolone was slowly injected. We succeeded in passing the NaviCath into the area of interest in all patients except 4 who had undergone previous surgery. When the procedure was over a sterile dressing was applied around the sacral hiatus. Subsequently, the patient was turned to the supine position and transferred to the recovery room. In the recovery room, the patient was monitored very closely for any potential complications or side effects.

\section{Statistical analysis}

A Wilcoxon ranking test was used to assess the clinical improvement in the NRS back, NRS leg, and ODI(\%) at 2 weeks and 3 months after PA. To determine prognostic predictors of PA among the clinical parameters and MRI findings, we compared patients' ages using a Mann-Whitney $U$ test as well as sex distribution, duration of symptoms, predominant symptom between back and leg pain, and presence of previous operation history, type and location of herniated disc, number of lesion levels, grade of nerve root compression, and the co-existence of spinal stenosis using Chi-squared test with Fisher's exact tests between successful and unsuccessful results for NRS and ODI. All statistical analyses were performed using the SPSS Version 12.0 statistical package (IBM Corporation, Armonk, NY). Results were considered statistically significant if $P<0.05$. 


\section{Results}

At 2 weeks after PA, significant improvement was observed in NRS back, NRS leg, and ODI compared with pretreatment, and was maintained until 3 months after treatment (Table 1). At pretreatment, there were no significant differences in age, sex ratio, NRS back, NRS leg, and ODI between successful and unsuccessful results (Table 2 ).

Among the 86 patients, successful outcomes at 2 weeks were obtained for the following: NRS back, 61
Table 1. Comparison of NRS and ODI between pretreatment and post treatment.

\begin{tabular}{|l|l|l|l|l|}
\hline $\begin{array}{l}\text { NRS } \\
\text { back }\end{array}$ & Pretreatment- 2 weeks & $5.43 \pm 2.11$ & $2.48 \pm 1.89$ & $P<0.001$ \\
\hline $\begin{array}{l}\text { NRS } \\
\text { leg }\end{array}$ & Pretreatment-3 months & $5.53 \pm 2.02$ & $2.91 \pm 2.12$ & \\
\hline PDI Peeks & $5.88 \pm 2.66$ & $3.12 \pm 2.48$ & \\
$(\%)$ & Pretreatment-3 months & $5.82 \pm 2.58$ & $2.91 \pm 2.41$ & \\
\hline
\end{tabular}

NRS back: Back pain score of Numeric rating scale NRS leg: Leg pain score of Numeric rating scale ODI: Oswestry disability score

Table 2. Comparison of clinical and MRI findings between patients with successful and unsuccessful results in NRS back.

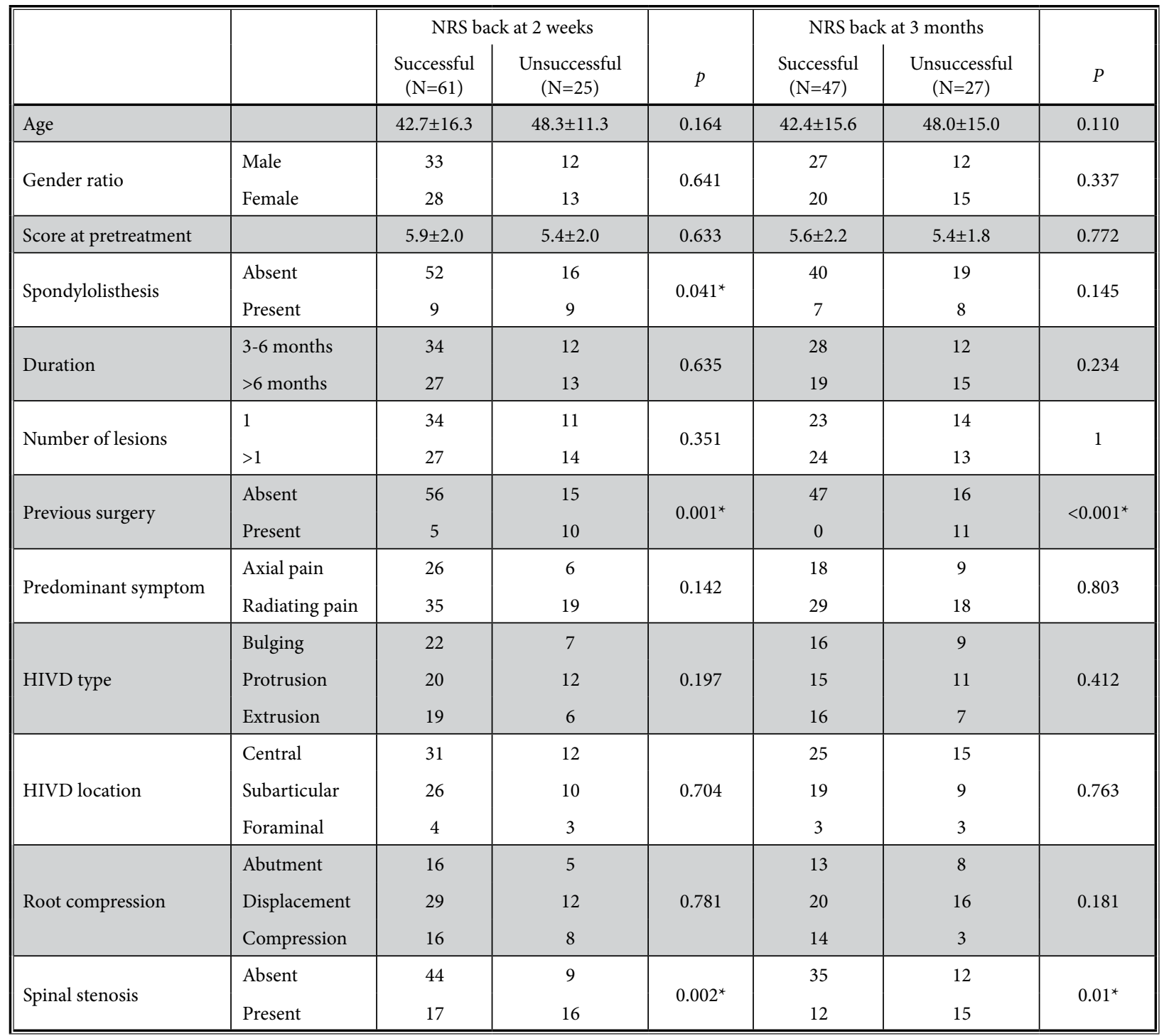

NRS back : Back pain score of Numeric rating scale

HIVD : herniaition of intervertebral disc.

Successful pain relief was described as $50 \%$ or more reduction of NRS. 
patients (70.9\%); NRS leg, 53 patients (61.6\%); and ODI, 61 patients $(70.9 \%)$. Nine patients with persistent pain after PA underwent back surgery and another 3 patients were lost during follow-up. Among the 74 patients followed up at 3 months, successful results were obtained for the following: NRS back, 47 patients (63.5\%); NRS leg, 44 patients (59.5\%); and ODI, 50 patients $(67.6 \%)$.

A significantly higher proportion of patients with a history of previous lumbar surgery showed unsuccessful results on NRS back, NRS leg, and ODI scores at 2 weeks and 3 months. Co-existence of spinal stenosis was associated with a significantly higher proportion of unsuccessful results in NRS back and ODI at 2 weeks and 3 months as well as NRS leg at 3 months. Patients with spondylolisthesis also showed a significantly higher proportion of unsuccessful results on their NRS and ODI scores at 2 weeks (Tables 2-4).

Ten of 86 patients complained of transient local pain in the lower back and needle insertion area. This pain was well controlled by analgesics. Only one pa-

Table 3. Comparison of clinical and MRI findings between patients with successful and unsuccessful results in NRS leg.

\begin{tabular}{|c|c|c|c|c|c|c|c|}
\hline & & \multicolumn{2}{|c|}{ NRS leg 2 weeks } & \multirow[b]{2}{*}{$\boldsymbol{p}$} & \multicolumn{2}{|c|}{ NRS leg 3 months } & \multirow[b]{2}{*}{$\boldsymbol{p}$} \\
\hline & & $\begin{array}{l}\text { Successful } \\
(\mathrm{N}=\mathbf{5 3})\end{array}$ & $\begin{array}{c}\text { Unsuccessful } \\
(\mathbf{N}=33)\end{array}$ & & $\begin{array}{l}\text { Successful } \\
\qquad(N=44)\end{array}$ & $\begin{array}{c}\text { Unsuccessful } \\
(\mathbf{N}=\mathbf{3 0})\end{array}$ & \\
\hline Age & & $44.9 \pm 15.2$ & $43.3 \pm 15.3$ & 0.683 & $44.1 \pm 14.9$ & $44.9 \pm 16.6$ & 0.881 \\
\hline \multirow{2}{*}{ Gender ratio } & Male & 30 & 15 & \multirow{2}{*}{0.377} & 26 & 13 & \multirow{2}{*}{0.237} \\
\hline & Female & 23 & 18 & & 18 & 17 & \\
\hline Score at pretreatment & & $6.1 \pm 2.5$ & $5.8 \pm 2.9$ & 0.599 & $5.9 \pm 2.3$ & $5.7 \pm 2.9$ & 0.897 \\
\hline \multirow[b]{2}{*}{ Spondylolisthesis } & Absent & 45 & 23 & \multirow[b]{2}{*}{0.108} & 37 & 22 & \multirow[b]{2}{*}{0.258} \\
\hline & Present & 8 & 10 & & 7 & 8 & \\
\hline \multirow{2}{*}{ Duration } & 3-6 months & 30 & 16 & \multirow{2}{*}{0.51} & 27 & 13 & \multirow{2}{*}{0.157} \\
\hline & $>6$ months & 23 & 17 & & 17 & 17 & \\
\hline \multirow{2}{*}{ Number of lesions } & 1 & 29 & 16 & \multirow{2}{*}{0.659} & 24 & 13 & \multirow{2}{*}{0.478} \\
\hline & $>1$ & 24 & 17 & & 20 & 17 & \\
\hline \multirow{2}{*}{ Previous surgery } & Absent & 48 & 23 & \multirow{2}{*}{$0.019^{*}$} & 42 & 21 & \multirow{2}{*}{$0.004^{*}$} \\
\hline & Present & 5 & 10 & & 2 & 9 & \\
\hline \multirow{2}{*}{ Predominant symptom } & Axial pain & 19 & 13 & \multirow{2}{*}{0.82} & 15 & 12 & \multirow{2}{*}{0.631} \\
\hline & Radiating pain & 34 & 20 & & 29 & 18 & \\
\hline \multirow{3}{*}{ HIVD type } & Bulging & 18 & 11 & \multirow{3}{*}{0.257} & 15 & 10 & \multirow{3}{*}{0.237} \\
\hline & Protrusion & 16 & 16 & & 13 & 13 & \\
\hline & Extrusion & 19 & 6 & & 16 & 7 & \\
\hline \multirow{3}{*}{ HIVD location } & Central & 26 & 17 & \multirow{3}{*}{0.947} & 22 & 18 & \multirow{3}{*}{0.175} \\
\hline & Subarticular & 23 & 13 & & 20 & 8 & \\
\hline & Foraminal & 4 & 3 & & 2 & 4 & \\
\hline \multirow{3}{*}{ Root compression } & Abutment & 15 & 6 & \multirow{3}{*}{0.344} & 11 & 10 & \multirow{3}{*}{0.549} \\
\hline & Displacement & 26 & 15 & & 21 & 15 & \\
\hline & Compression & 12 & 12 & & 12 & 5 & \\
\hline \multirow{2}{*}{ Spinal stenosis } & Absent & 35 & 18 & 0207 & 32 & 15 & $0046 t$ \\
\hline & Present & 18 & 15 & 0.207 & 12 & 15 & 0.040 \\
\hline
\end{tabular}

NRS leg : Leg pain score of Numeric rating scale HIVD : herniaition of intervertebral disc.

Successful pain relief was described as $50 \%$ or more reduction of NRS. 
Pain Physician: May/June 2012; 15:213-221

Table 4. Comparison of clinical and MRI findings between patients with successful and unsuccessful results in ODI.

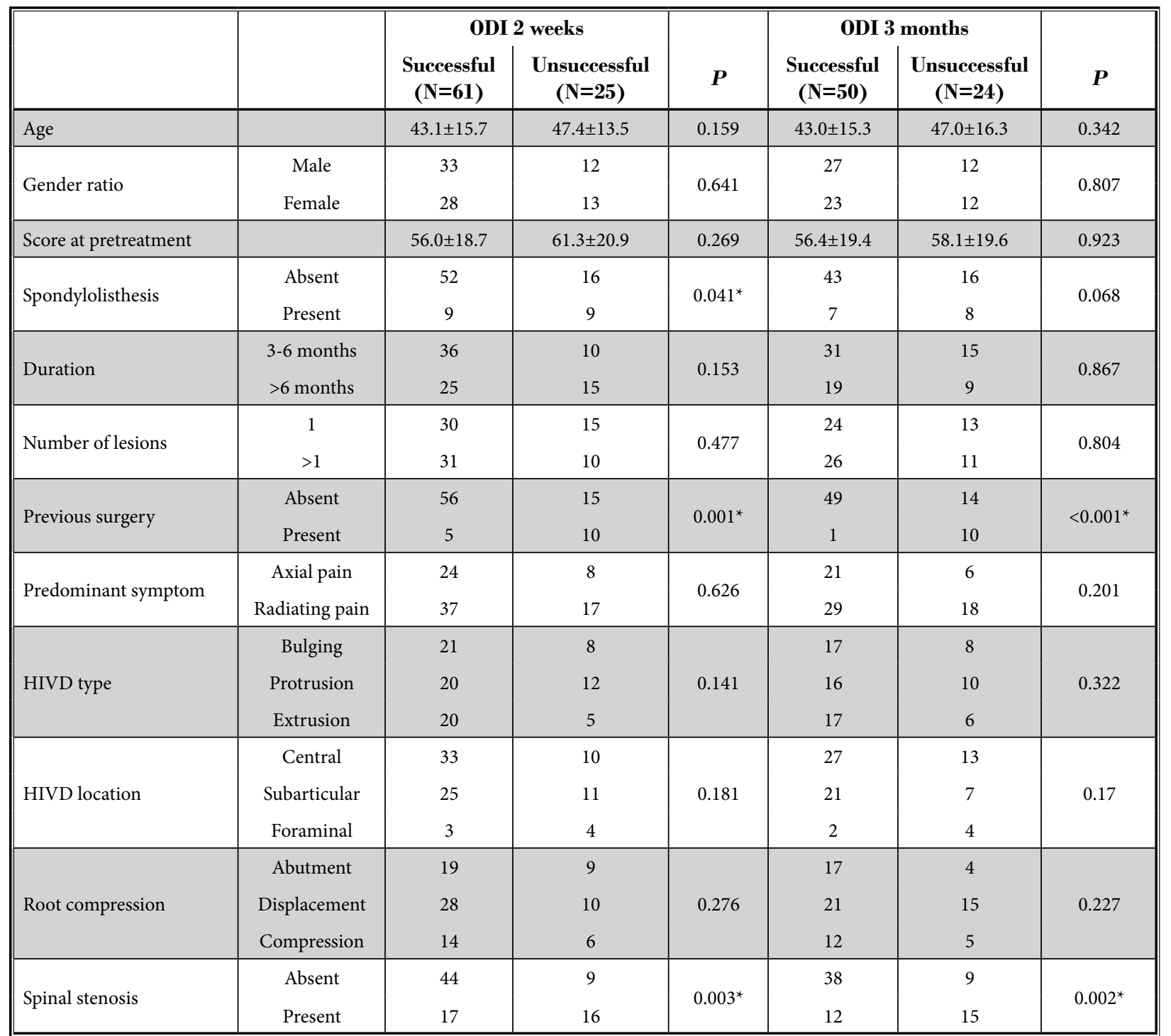

ODI : Oswestry disability score

HIVD : herniaition of intervertebral disc.

Successful functional improvement of ODI was defined as $40 \%$ or more reduction of ODI.

tient experienced a relatively severe complication of headache and transient seizure-like motions after the procedure. These symptoms disappeared without any specific treatment. This patient had a previous history of several episodes of generalized seizures and had taken antiepileptic drugs for 5 years. Among the 9 patients who underwent surgery within the 3-month follow-up period, only one patient underwent interbody fusion surgery and 8 patients underwent microscopic open discectomy. All of them showed satisfactory re- sults in pain reduction and functional improvement after surgery.

\section{Discussion}

PA has been used for the treatment of chronic pain that is intractable to other conservative management and has been shown to have good clinical efficacy (18). PA demonstrates superior effectiveness compared to not only physical therapy, (29) but also caudal epidural steroid injections for the treatment of chronic lower 
back and leg pain $(23,30)$. This is because PA is able to eliminate adhesions or fibrous tissue that might prevent the spread of injected medications into the specific lesion site, and allows placement of the catheter tip within the target areas. Consequently, this enables the application of an adequate concentration of the steroid or other solution to the appropriate target area (31).

Previous reports regarding the efficacy of PA used the Racz catheter. Our study used NaviCath with normal saline. NaviCath has a steerable catheter and an atraumatic tip, and is different from the Racz catheter that has a catheter and spring tip that cannot be steered. This property of NaviCath enables the physician to place the catheter tip near the nerve root sheath and deliver pain medication more precisely. This might lead to performing mechanical adhesiolysis more easily.

Manchikanti et al (22) compared the clinical efficacy among 3 different treatment groups: Group I consisted of a control group not undergoing adhesiolysis with normal saline; Group II consisted of patients undergoing adhesiolysis with normal saline; and Group III consisted of patients undergoing adhesiolysis with $10 \%$ hypertonic saline. Approximately $70 \%$ of patients in Group III , $60 \%$ of patients in Group II, and $0 \%$ of patients in Group I showed significant improvement in the Visual Analog Scale (VAS) and ODI at 12-months follow-up. Another comparative study showed that most patients achieved significant reduction of the VAS after PA and the percentage of patients with VAS improvement did not differ whether the injectate included hypertonic saline or normal saline (32). These studies suggested that appropriate mechanical adhesiolysis with normal saline could also be an effective treatment and obtained comparable results to adhesiolysis with hypertonic saline in chronic refractory back pain. Thus, it was assumed that precise placement of the catheter tip by NaviCath, which would allow for appropriate adhesiolysis, did not require hypertonic saline, which could cause serious adverse effects $(16,33)$.

Studies on the clinical efficacy of PA have reported that PA shows more significant pain reduction and better functional outcomes than do medication, physical therapy, and an exercise program $(29,32,34)$. In one study, about $49 \%$ of the patients that failed to achieve clinical improvement by other conservative treatments showed a significant reduction of their pain score at 3 months, while $43 \%$ achieved pain reduction at 6 months, and $49 \%$ at 12 months (32). Few studies have compared the efficacy of PA with that of epidural steroid injection. Two reports have identified that clinical outcomes of PA are better than caudal epidural injections in patients with spinal stenosis and lumbar postsurgery syndrome $(23,30)$. But among 3 epidural injection techniques such as caudal, interlaminar, or transforaminal approach, the transforaminal approach offered better clinical effectiveness than the caudal or interlaminar approaches because it allowed the injectate to spread directly into the ventral epidural space (35-37). The implication of our study was that about $60-70 \%$ at 2 weeks and $55-60 \%$ at 3 months of the patients refractory to transforaminal epidural injection, assumed to be the most effective among epidural injection methods, could achieve significant clinical results.

In a clinical setting, PA is usually performed for patients who fail to improve clinically after an epidural steroid injection and is rarely performed for patients who have not first received an epidural injection. We assumed that PA was not required for patients who were responsive to an epidural injection since epidural injection is a simpler and less expensive procedure than PA. Therefore, we aimed to evaluate PA efficacy in patients who were unresponsive to transforaminal epidural injection, instead of comparing the clinical efficacy between PA and epidural injection.

To the best of our knowledge, no studies have assessed the clinical or laboratory factors related to the efficacy of PA. In our study, a significant relationship was not found between MRI characteristics of L-HIVD and the clinical efficacy of PA. However, the co-existence of spinal stenosis, spondylolisthesis, or previous surgical history was related to poorer outcomes. It has been reported that patients with spinal stenosis or postsurgery syndrome showed a worse response to epidural steroid injection than do L-HIVD patients $(32,38)$. Spinal stenosis and postsurgery syndrome were associated with irreversible changes such as epidural fibrosis, scarring, and hypertrophied lateral recess and ligament, which might render the nerve root refractory to management by the local application of steroids $(31,36,38)$. These features were assumed to be more severe and irreversible than L-HIVD and were often associated with scar formation or bony hypertrophy, which interfered with advancement of the catheter or injectates into the ventral epidural space. Spondylolisthesis also leads to not only segmental instability but also a diminished cross-sectional area of the vertebral canal, apparent thickening and buckling of the ligamentum flavum, or hypertrophy of adjacent facet joints, thus contributing to spinal stenosis (39). These structural characteristics could explain why patients with spinal stenosis, spondylolisthesis, or 
postsurgery syndrome showed poorer outcomes than those without.

PA may cause epidural hematoma, infection, excessive intraspinal and intracranial pressures, and increased intraocular pressures with resultant visual deficiency. The most troubling complications are mainly related to dural puncture, spinal cord compression, intravascular injection, and administration of high volumes of fluids, potentially resulting in excessive epidural hydrostatic pressures, death, and brain damage $(18,19,32,40)$. In our patient group, one patient who was receiving antiepileptic treatment experienced the serious complication of transient seizure-like motions. An elevated intraspinal pressure or intrathecal administration of injectate might have triggered generalized seizure in this high risk patient.

There were several limitations in this study. First, the follow-up period was relatively short. This might weaken the power of this study. Second, this study was retrospective in design. Despite these limitations, we suggested the important clinical points. We performed the PA for patients refractory to transforaminal epi- dural injection and showed the positive results. In the clinical setting, many patients refractory to epidural injection were regarded as failures of conservative management and consequently, underwent the surgical procedure. This study could suggest the ability of PA to be another treatment strategy for patients who could not accomplish successful results by epidural injections and might negate the need for lumbar surgery.

\section{Conclusion}

PA was effective for pain reduction and functional improvement in patients with chronic lower back or leg pain due to L-HIVD who did not respond to other conservative treatments including transforaminal epidural injection. A history of previous surgery and the co-existence of spinal stenosis or spondylolisthesis were revealed to be poor prognostic predictors of PA.

\section{Acknowledgements}

This study was supported by Wooridul spine foundation.

\section{References}

1. Manchikanti L, Rivera JJ, Pampati V, Damron KS, Beyer CD, Brandon DE, Wilson SR. Spinal endoscopic adhesiolysis in the management of chronic low back pain: A preliminary report of a randomized, double-blind trial. Pain Physician 2003; 6:259-267.

2. Anderson SR, Racz GB, Heavner J. Evolution of epidural lysis of adhesions. Pain Physician 2000; 3:262-270.

3. Manchikanti L, Singh V, Kloth D, Slipman CW, Jasper JF, Trescot AM, Varley KG, Atluri SL, Giron C, Curran MJ, Rivera J, Baha AG, Bakhit CE, Reuter MW. Interventional techniques in the management of chronic pain: Part 2.o. Pain Physician 2001; 4:24-96.

4. Cooper RG, Freemont AJ, Hoyland JA, Jenkins JP, West CG, Illingworth KJ, Jayson MI. Herniated intervertebral discassociated periradicular fibrosis and vascular abnormalities occur without inflammatory cell infiltration. Spine (Phila Pa 1976) 1995; 20:591-598.

5. Heavner JE, Bosscher HA, Wachtel MS. Cell types obtained from the epidural space of patients with low back pain/ radiculopathy. Pain Pract 2009; 9:167172.

6. Kobayashi S, Baba H, Uchida K, Kokubo Y, Kubota C, Yamada S, Suzuki Y, Yoshizawa $H$. Effect of mechanical com- pression on the lumbar nerve root: localization and changes of intraradicular inflammatory cytokines, nitric oxide, and cyclooxygenase. Spine (Phila Pa 1976) 2005; 30:1699-1705.

7. $\mathrm{Ng} \mathrm{L}$, Chaudhary N, Sell P. The efficacy of corticosteroids in periradicular infiltration for chronic radicular pain: A randomized, double-blind, controlled trial. Spine (Phila Pa 1976) 2005; 30:857-862.

8. Parr AT, Diwan S, Abdi S. Lumbar interlaminar epidural injections in managing chronic low back and lower extremity pain: A systematic review. Pain Physician 2009; 12:163-188.

9. Vad VB, Bhat AL, Lutz GE, Cammisa F. Transforaminal epidural steroid injections in lumbosacral radiculopathy: $A$ prospective randomized study. Spine ( $\mathrm{Ph}$ ila $\mathrm{Pa}$ 1976) 2002; 27:11-16.

10. Rosenberg SK, Grabinsky A, Kooser C, Boswell MV. Effectiveness of transforaminal epidural steroid injections in low back pain: A one year experience. Pain Physician 2002; 5:266-270.

11. Manchikanti L, Singh V, Cash KA, Pampati $V$, Datta $S$. Preliminary results of a randomized, equivalence trial of fluoroscopic caudal epidural injections in managing chronic low back pain: Part 3--Post surgery syndrome. Pain Physician 2008; 11:817-831.
12. Manchikanti L, Singh V, Cash KA, Pampati V, Datta S. Management of pain of post lumbar surgery syndrome: Oneyear results of a randomized, doubleblind, active controlled trial of fluoroscopic caudal epidural injections. Pain Physician 2010; 13:509-521.

13. Delport EG, Cucuzzella AR, Marley JK, Pruitt CM, Fisher JR. Treatment of lumbar spinal stenosis with epidural steroid injections: A retrospective outcome study. Arch Phys Med Rehabil 2004; 85:479-484.

14. Wilson-MacDonald J, Burt G, Griffin D, Glynn C. Epidural steroid injection for nerve root compression. A randomised, controlled trial. J Bone Joint Surg $\mathrm{Br}$ 2005; 87:352-355.

15. Rosen CD, Kahanovitz N, Bernstein R, Viola K. A retrospective analysis of the efficacy of epidural steroid injections. Clin Orthop Relat Res 1988: 270-272.

16. Manchikanti L, Bakhit CE. Percutaneous lysis of epidural adhesions. Pain Physician 2000; 3:46-64.

17. Manchikanti L, Singh V, Bakhit CE, Fellows B. Interventional techniques in the management of chronic pain: Part 1.o. Pain Physician 2000; 3:7-42.

18. Chopra P, Smith HS, Deer TR, Bowman RC. Role of adhesiolysis in the management of chronic spinal pain: A systemat- 
ic review of effectiveness and complications. Pain Physician 2005; 8:87-100.

19. Trescot AM, Chopra P, Abdi S, Datta S Schultz DM. Systematic review of effectiveness and complications of adhesiolysis in the management of chronic spinal pain: An update. Pain Physician 2007; 10:129-146.

20. Manchikanti L SP, Singh V, Schultz DM, Vilims BD, Jasper JF, Kloth DS, Trescot AM, Hansen HC, Falasca TD, Racz GB, Deer T, Burton AW, Helm S, Lou L, Bakhit CE,Dunbar EE, Atluri SL, Calodney AK, Hassenbusch S, Feler CA. Evidence-based practice guidelines for interventional techniques in the management of chronic spinal pain. Pain Physician 2003; 6:3-80.

21. Viesca C, Racz G, Day M. Spinal techniques in pain management: Lysis of adhesions. Anesthesiol Clin North America 2003; 21:745-766.

22. Manchikanti L RJ, Pampati V, Damron KS, MCManus CD, Brandon DE, Wilson SR. One day lumbar epidural adhesiolysis and hypertonic saline neurolysis in treatment of chronic low back pain: A randomized double blind trial. Pain Physician 2004; 7:177-186.

23. Manchikanti L, Singh V, Cash KA, Pampati $V$, Datta S. A comparative effectiveness evaluation of percutaneous adhesiolysis and epidural steroid injections in managing lumbar post surgery syndrome: A randomized, equivalence controlled trial. Pain Physician 2009; 12:E355-E368.

24. Choi SJ, Song JS, Kim C, Shin MJ, Ryu DS, Ahn JH, Jung SM, Park MS. The use of magnetic resonance imaging to predict the clinical outcome of non-surgical treatment for lumbar intervertebral disc herniation. Korean J Radiol 2007; 8:156163.
25. Jeon CH, Kim DJ, Kim SK, Lee HM, Park HJ. Validation in the cross-cultural adaptation of the Korean version of the Oswestry Disability Index. J Korean Med Sci 2006; 21:1092-1097.

26. Manchikanti L, Cash KA, McManus CD, Pampati V, Abdi S. Preliminary results of a randomized, equivalence trial of fluoroscopic caudal epidural injections in managing chronic low back pain: Part 4--Spinal stenosis. Pain Physician 2008; 11:833-848.

27. Cooper G, Lutz GE, Boachie-Adjei O, Lin $J$. Effectiveness of transforaminal epidural steroid injections in patients with degenerative lumbar scoliotic stenosis and radiculopathy. Pain Physician 2004; 7:311-317.

28. Fairbank JC, Pynsent PB. The Oswestry Disability Index. Spine 2000; 25:29402952; discussion 2952.

29. Veihelmann A, Devens C, Trouillier H Birkenmaier C, Gerdesmeyer L, Refior HJ. Epidural neuroplasty versus physiotherapy to relieve pain in patients with sciatica: A prospective randomized blinded clinical trial. J Orthop Sci 2006; 11:365-369.

30. Manchikanti L, Cash KA, McManus CD Pampati $V$, Singh $V$, Benyamin R. The preliminary results of a comparative effectiveness evaluation of adhesiolysis and caudal epidural injections in managing chronic low back pain secondary to spinal stenosis: A randomized, equivalence controlled trial. Pain Physician 2009; 12:E341-E354.

31. Epter RS, Helm S, Hayek SM, Benyamin RM, Smith HS, Abdi S. Systematic review of percutaneous adhesiolysis and management of chronic low back pain in post lumbar surgery syndrome. Pain Physician 2009; 12:361-378.
32. Heavner J, Racz G, Raj P. Percutaneous epidural neuroplasty. Prospective evaluation of $0.9 \% \mathrm{NaCl}$ versus $10 \% \mathrm{NaCl}$ with or without hyaluronidase. Reg Anesth Pain Med 1999; 24:202-207.

33. Kim RC, Porter RW, Choi BH, Kim SW. Myelopathy after the intrathecal administration of hypertonic saline. Neurosurgery 1988; 22:942-945.

34. Manchikanti L, Pampati V, Fellows B, Rivera J, Beyer CD, Damron KS. Role of one day epidural adhesiolysis in management of chronic low back pain: A randomized clinical trial. Pain Physician 2001; 4:153-166.

35. Ackerman WE, Ahmad M. The efficacy of lumbar epidural steroid injections in patients with lumbar disc herniations. Anesth Analg 2007; 104:1217-1222.

36. Lee JH, An JH, Lee SH. Comparison of the effectiveness of interlaminar and bilateral transforaminal epidural steroid injections in treatment of patients with lumbosacral disc herniation and spinal stenosis. Clin J Pain 2009; 25:206-210.

37. Lee JH, Moon J, Lee SH. Comparison of effectiveness according to different approaches of epidural steroid injection in lumbosacral herniated disk and spinal stenosis. J Back Musculoskelet Rehabil 2009; 22:83-89.

38. Rivest C, Katz JN, Ferrante FM, Jamison RN. Effects of epidural steroid injection on pain due to lumbar spinal stenosis or herniated disks: a prospective study. Arthritis Care Res 1998; 11:291-297.

39. Kalichman L, Hunter DJ. Diagnosis and conservative management of degenerative lumbar spondylolisthesis. Eur Spine J 2008; 17:327-335.

40. Manchikanti L. Role of neuraxial steroids in interventional pain management. Pain Physician 2002; 5:182-199. 
